\title{
Finger Vein Detection using Gabor Filter, Segmentation and Matched Filter
}

\author{
Poonam Dabas \\ Computer Science Department \\ UIET,Kurukshetra University \\ Kurukshetra, India \\ poonamdabas.kuk@gmail.com
}

\author{
Amandeep Kaur \\ Computer Science Department \\ UIET, Kurukshetra University \\ Kurukshetra, India \\ amandeepg5s@gmail.com
}

\begin{abstract}
This paper propose a method of personal identification based on finger-vein patterns. An image of a finger captured by the web camera under the IR light transmission contains not only the vein pattern itself; but also shade produced by various thickness of the finger muscles; bones; and tissue networks surrounding the vein. In this paper; we intro-duce preliminary process to enhance the image quality worsened by light effect and noise produced by the web camera; then segment the vein pattern by using adaptive threshold method and matched them using improved template matching. The main purposes of this paper are to investigate finger-vein technology; the applicable fields and whether finger-vein detection can solve the problems fingerprint detection imposes in certain industries.
\end{abstract}

Keywords: Finger-vein detection, Gabor filter, filter, pattern recognition.

\section{INTRODUCTION}

Smart recognition [1][2] of human identity for security and control is a global issue of concern in our world today. The financial losses due to identity theft can be severe; and the integrity of security systems compromised. The automatic authentication systems for control have found application in criminal identification; autonomous vending and automated banking among others. Amongst the more authentication systems that have been proposed and implemented; finger vein biometrics is emerging as the foolproof method of automated personal identification. The Finger vein is a unique physiological biometric for identifying individuals based on the physical characteristics and attributes of the vein patterns in the human finger. This is a fairly recent technological advance in the field of biometrics that is being applied to different fields such as medical; financial; law enforcement facilities and other applications where high levels of security or privacy is very important. The technology is impressive because it requires only small; relatively cheap single-chip design; and has a very fast identification process that is contact-less and of higher accuracy when compared with other identification biometrics like fingerprint; iris; facial and others. This much accuracy rate of finger vein is not unconnected with the fact that finger vein patterns are virtually impossible to forge thus it has become one of the fastest growing new biometric technology that is quickly finding its way from research labs to commercial development.
Vein image is obtained from the infrared image collection. Research has proved that the absorption of infrared light in human tissues is comparatively low, i.e. the infrared light has more penetrating ability for human body. Though the hemoglobin has a strong absorption of infrared light, experiment proves that quasi-infrared light can make good imaging of subcutaneous blood vessel of $0-1 \mathrm{~cm}$ depth [3].

Due to the poor infrared image quality, image enhancement and Region of Interest (ROI) techniques [4] are introduced in data collection module. After the optimized image is obtained, the vein pattern is derived according to segmentation, skeletonization refinement and feature definition procedures, and then the pattern is saved to the database as template in the registration mode. In the verification mode, the pattern is compared with the templates in the database and result can be obtained. Since vein sampling is from subcutaneous image, it is hard to be destroyed and stolen so that it can be an ideal candidate of high-level verification technique.

In this paper, we studied the vein identification [6] on trial and discovered that there were several difficulties:

a. The image grabbed by the common web camera consists of salt and pepper noise and the gray level distribution among different trial is not the same, because the web camera always does the brightness adjustment. 
b. On normal conditions, gray scale discrimination of vein image is very small. We need a good threshold segmentation to get the effective binary image that provides sufficient finger vein information.

c. The pressure given on our finger will cause the vein in-side shrink or changed. So, we need to build a "lessstrict" finger slot to let the user's finger in a "relax" condition.

First, a new approach for personal identification that utilizes simultaneously acquired finger-vein and finger

[5]. Therefore, the acquired images are first subjected to pre-processing steps that include:

\section{Segmentation of ROI,}

2. Translation and orientation alignment, and

3. Image enhancement to extract stable/reliable vascular Patterns.

The enhanced and normalized ROI images [7] are employed for feature extraction. The key objective while segmenting the ROI is to automatically normalize the region in such a way that the image variations; caused by the interaction of the user with the imaging device; can be minimized. The order to make the identification process more effective and efficient; it is necessary to construct a coordinate system that is invariant or robust (or nearly) to such variations. This is judicious to associate the coordinate system with the Finger itself since we are seeking the invariance corresponding to it. As a result two webs are utilized as the reference points/line to build up the coordinate system i.e. web among the index finger and middle finger together with the web between the ring finger and little finger. These web points are easily identified in touch-based imaging (using pegs) but should be automatically generated for contactless imaging [8].

The acquired Finger images are first binarized, so that we are able to separate the Finger region from the background region. It is followed by the estimation of the distance from centre position of the binarized Finger to the boundary of Finger. surface (texture) images is presented. The experimental results illustrate significantly improved performance that cannot be achieved by any of these images employed individually.

\section{Finger-Vein Image Pre-processing}

The acquired images are noisy with rotational and translational variations resulting from unconstrained imaging.

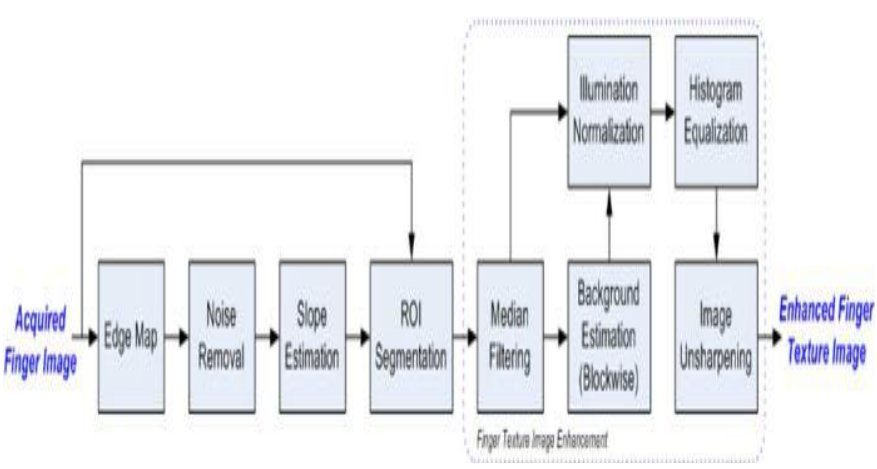

Figure 1. Block diagram illustrating key steps employed for the preprocessing of acquired finger texture images from a webcam.

\section{Recognition}

The same as fingerprint recognition [9], finger vein recognition also contains image pre-processing, feature extraction and matching. The image normalization, finger vein segmentation, thinning is all images preprocessing work. Then acquisition time; light intensity of acquisition environment; thickness of each finger; and intensity distribution of finger vein images are all different; hence normalization of image size and gray scale is indispensable in order to feature extraction and classification.

Finger vein contains ridge and valley lines. In addition to show paragraphs must be indented. Then all paragraphs must be justified; both left-justified and right-justified irregular shape in the minutiae and singularity regions; the ridge and valley lines show continuous and smooth change in most regions. The repeated line tracking method gives a promising result in finger-vein identification: The idea is to trace the veins in the image by chosen directions according to predefined probability in the horizontal and vertical orientations; and the starting seed is randomly selected; the whole process is repeatedly done for a certain number of times. 
This process of improving the quality of a digitally damage by manipulating the image with software. This is quite easy; for example to make an image lighter or darker or to increase or decrease contrast.

In paper; Gabor filter was used to enhance finger vein images. In this paper, the systematical development of a new approach for the finger-vein feature extraction using Gabor filters is introduced. And in addition; we also investigate a new feature extraction approach using matched filters as the matched filters have been successfully utilized for the enhancement of retinal features in [10].

The Gabor filters are inspired by the multichannel processing of visual information in the biological model of human visual system and are known to achieve the maximum possible joint resolution in the spatial and spatial-frequency domains [11], which have been effectively utilized by researchers to develop object segmentation paradigm. This paper; proposed the framework for the finger vein feature extraction using multi orientation Gabor filters. We will be using GABOR Filter, Median Filter and Repeated Line. Tracking method for recognition.

\section{Conclusions}

This paper will present a complete and fully automated Finger image matching and Finger subsurface features, i.e., from Finger-vein images. This will present a new algorithm for the Finger-vein identification; which can more reliably extract the Finger-vein shape features and achieve much higher accuracy than previously proposed Finger-vein identification approaches. The Finger -vein matching scheme will work more effectively in more realistic scenarios and leads to a more accurate performance; as will be demonstrated from the experimental results. At last; examine a complete and fully automated approach for the identification of low resolution Finger-surface for the performance improvement.

\section{References}

[1] Jain, A.; Ross, A.; Prabhakar, S. An introduction to biometric recognition. IEEE Trans. Circ. Syst. Video Tech. 2004, 14, 4-20.

[2] Jain, A.K.; Fengs, J.; Nandakumar, K. Fingerprint matching. Computer 2010, 43, 36-44.
[3] Guo, Z.; Zhang, D.; Zhang, L.; Zuo, W. Palm print verification using binary orientation co-occurrence vector. Patt. Recogn. Lett. 2009, 30, 1219-1227.

[4] Ito, K.; Nakajima, H.; Kobayashi, K.; Aoki, T.; Higuchi, T. A fingerprint matching algorithm using phase-only correlation. IEICE Trans. Fundament. Electron. Commun. Comput. Sci. 2004, E87-A, 682691.

[5] Zhang, L.; Zhang, L.; Zhang, D.; Zhu, H. Ensemble of local and global information for finger-knuckle-print recognition. Patt. Recogn. 2011, 44, 1990-1998.

[6] Miura, N.; Nagasaka, A.; Miyatake, T. Feature extraction of finger-vein patterns based on repeated line tracking and its application to personal identification. Mach. Vision Appl. 2004, 15, 194-203.

[7] Yanagawa, T.; Aoki, S.; Ohyama, T. Human finger vein images are diverse and its patterns are useful for personal identification. MHF Preprint Ser. 2007, 12, 1 7.

[8] Y. Yang and M. Levine, "The Background Primal Sketch: An Approach for Tracking Moving Objects," Machine Vision and Applications, vol. 5, pp. 17-34, 1992.

[9] S. Chaudhuri, S. Chatterjee, N. Katz, M. Nelson, and M. Goldbaum, "Detection of blood vessels in retinal images using two-dimensional matched filters," IEEE Trans. Med. Imag., vol. 8, no. 3, pp. 263-269, Sep. 1989.

[10] J. G. Daugman, "High confidence visual recognition of persons by a test of statistical independence," IEEE Trans. Pattern Anal. Mach. Intell., vol. 15, no. 11, pp. 1148-1161, Nov. 1993.

[11] Y. Kuno, T. Watanabe, Y. Shimosakoda, and S. Nakagawa, "Automated Detection of Human for System. 